\title{
A Socially Assistive Robot for People with Motor Impairments
}

\author{
Manuel Pinheiro and Estela Bicho \\ Dept. of Industrial Electronics \\ Minho University \\ Campus de Azurem \\ 4800-058 Guimarães (PORTUGAL) \\ manuelspinheiro@gmail.com and estela.bicho@dei.uminho.pt
}

\begin{abstract}
We present a control architecture for nonverbal HRI that allows an anthropomorphic assistant robot with a pro-active and anticipatory behaviour. The control architecture coordinates action and goal coordination between a motor impaired human and the robot as a dynamic process that combines contextual cues, shared task knowledge, and predicted outcomes of the human behaviour. The control architecture is formalized through a coupled system of dynamic neural fields, representing a distributed network of local but connected neural populations with specific functionalities. Each subpopulation encodes relevant information about action means, goals, and context as selfsustained activation patterns. These patterns are triggered by the input and evolve continuously in time under the influence of recurrent interactions. The architecture is validated in an assistive task where the robot acts as an assistant of a person with motor impairments. We show that the context dependent mapping from action observation onto appropriate complementary actions allows the robot to cope with dynamically changing situations. This includes adaptation to different users and mutual compensation of physical limitations.
\end{abstract}

Index Terms-Socially Assistive Robotics; Mirror Neurons; Dynamic Neural Fields

\section{INTRODUCTION}

One of the current challenges in the robotics research field is how to develop autonomous robots able to assist people in a human-like way (reviews e.g. [1]-[5]). It is proved that Humans prefer to interact with machines in the same way that they interact with other people [6]. In order to guarantee user acceptance, a personal assistant robot should have social and cognitive capacities for natural and efficient humanrobot interaction. During assistive tasks, Humans continuously monitor the actions of each other, interpreting their underlying motor intentions, and predicting their concomitant outcomes. These predictions are used select the adequate action, that complements and coordinates with what is observed [7], [8]. For instance, imagine the task of assisting a person during a meal. By observing the way the other person grasps an object, e.g. a bottle of juice, allows us foresee the ultimate

\section{Master Thesis}

Portuguese chapter of IEEE EMBS

$3^{\text {rd }}$ Portuguese Meeting in Bioengineering, February 2013

University of Minho, Braga, Portugal goal of that action. By predicting the goal of the complete action at the time of grasping, allows the observer to timely grasp and hold out the glass, or to prepare to receive the bottle. This kind of nonverbal communication is considered essential for action fluency. Reading the nonverbal cues inherent to every behaviour favours the action transparency, the interaction robustness and bridges physical and cognitive (dis)abilities [9].

In this work we present the preliminary results of an ongoing work that aims the development of a socially aware robot capable of helping humans with motor or cognitive disabilities. In our approach, the robot integrates in its cognitive structure recent experimental and theoretical findings about the neurocognitive mechanisms underlying perception and action in social contexts (e.g. [10]-[13], for a review see [7], [14]).

Recent finding have put in evidence that human and nonhuman primates may possess a mechanism for mirroring observed actions [10], [15]. This mechanism seems to be tuned only for goal-directed actions and enables the observer to match the observed action into his/her own motor repertoire. The mechanism is called the Mirror Neuron System (MNS) and is thought to be the basic mechanism for action understanding and goal inference within the brain. Experiments with primates and human subjects also suggest that these neurons have different degrees of specializations, and code different motor aspects of a goal directed action. There are populations of mirror neurons that only fire when a specific motor act is observed, whereas others have a broader spectrum of activation. These observations are in agreement with the theory that there exist motor neurons that code the means in which the action is performed; likewise there are others that are responsible for action goal representation (for an general overview on the subject see [16]). Another important trait of the MNS is that it enables action representation even when both agents have marked morphological differences [17]. This is also a clear evidence of goal representation within the MNS, which may resonant through the observer motor repertoire endowing him/her with a real action understanding mechanism. The goal seems to be the most relevant part of the action [18]. All these evidences are paramount milestones and allow us to apply the model of goal inference based on motor resonance in joint tasks, even when the teammates have 


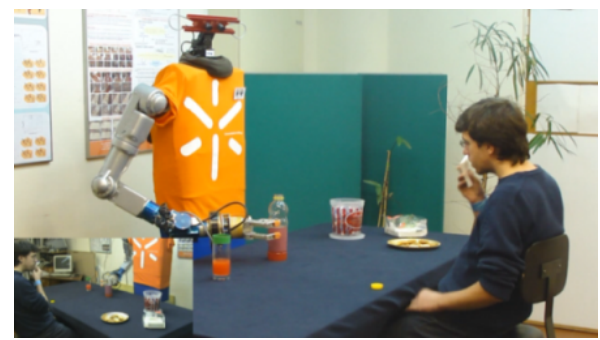

Fig. 1. The anthropomorphic robot acts as a pro-active personal assistant of a person, with motor impairments, that wishes to drink.

dissimilar embodiment like humans and robots. Nevertheless, the simulation of the motor behaviour of one's partner may not yield a full understanding of the action goal, because the same goal-direct action may have a different underlying goal depending on the action context. Thus, it is necessary to integrate additional contextual cues. In our previous work we have applied a dynamic neural field model of action understanding and complementary action selection that implement these neuro-cognitive principles. This dynamic field model was tested in a joint construction task; human and robot assemble a toy knowing the construction plan [19], [20]. The model consists of a distributed network of local pools of neurons with specific functionalities. Self-stabilized activity patterns in these populations represent potential goals; context and potential action means to pursue the goals. Observed objectdirected motor acts (e.g., grasping) along with contextual cues may trigger the propagation of activity through interconnected neural populations that constitute a learned chain of motor primitives directed towards a specific goal (e.g., reaching-grasping-placing at a particular position). Based on that excitatory chain of events the robot selects the proper complementary behaviour.

The paper is organized as follows: Section II introduces assistive task scenario and the robot platform. Section III gives an overview about the cognitive control architecture and presents the basic concepts of the dynamic field framework used to formalize and implement the architecture. The results of the human-robot interactions are described in section IV. The paper ends with the conclusions in section $\mathrm{V}$.

\section{Human-Robot Assistive Task Description}

In this work we have considered one of the most elementary daily tasks done by humans. We have constructed an interaction scenario where the robot helps a human with physical limitations to drink (Fig. 1).

The interaction scenario is constituted by two objects, a bottle and a glass, placed on a table. Although apparently simple, it is complex enough to show the impact of intention understanding on action selection, and how this can enable the robot to achieve user adaptation. Due to both agents' physical limitations, one has two workspaces: the human workspace (HWS); and the robot workspace (RWS). In addition, the objects can be placed on the table with different arrangements,

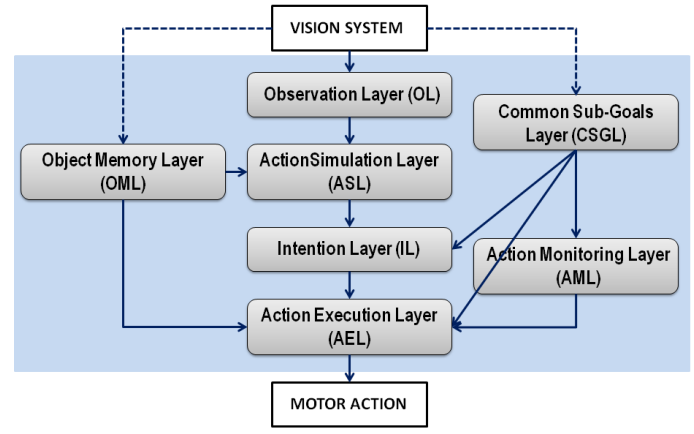

Fig. 2. The multi-layered cognitive control architecture for joint action and action monitoring

have different states, and orientations. Namely, the bottle can be closed or open; and the glass can be empty upright or inverted and full or empty. Based on the objects' initial state and disposition, the number and the nature of the subtasks to achieve the final joint action goal is different. The cooperation between the teammates is biased by the objects disposition, which may require handing over objects to one another. The main assumption in this interaction scenario is that the human user has a motor impairment that prevents him/her to perform the task alone. This assumption in addition to the fact that the robot does not have enough dexterity in its hand to open the bottle; is physically unable to grasp such a small object. The human is the only agent capable of removing the stopper/cup, but due to his/her motor impairment he/she cannot open the bottle alone and needs the robot's help to remove the cup. Thus, both agents are compelled to cooperate with each other in order to bridge and compensate their mutual motor limitations and fulfil the joint action successfully. The anthropomorphic robot was built in our lab [21]. It is constituted by a stationary torus on which a 7 DOFs AMTEC arm (Schunk GmbH) with a three finger dexterous gripper, and a stereo camera head are mounted. A speech synthesizer (Microsoft Speech SDK 5.1) is used to assure the verbal communication with the user. The visual information is provided by the camera system, and combines colour-based segmentation with template matching derived from earlier learning examples [22]. This technique is also used for the classification of object-directed, static hand postures such as grasping and communicative gestures such as pointing or demanding an object. The arm-hand system control is achieved by a global planning method in posture space, that allows the generation of smooth and human-like movements [23].

\section{Cognitive Control Architecture IMPLEMENTATION}

The robot's cognitive control architecture is inspired in the neuro-cognitive mechanisms underlying perception, reasoning and action in a social context, and is schematically presented in Fig. 2.

The architecture can be seen as a network of interconnected pools of neurons, organized in different layers. Each layer 
is responsible for coding a different type of information and enables the implementation of a dynamic process of action simulation, goal inference, action monitoring and complementary action selections [19]. The visual information produces activation patterns in specialized pools of neurons within the Object Memory Layer (OML), the Common Sub-Goals Layer (CSGL), and the Observation Layer (OL). In the OML specific pools of neurons represent the objects of interest in terms their general state and position within the agents' workplaces; the CSGL enable the robot to have an internal representation of the achieved intermediate goals and the sub-goals that still have to be accomplished; and in the OL the motor acts observed by the robot are translated into motor primitives. The patterns of activation that begin to emerge may become selfsustainable. Once this occurs, these elicit activation patterns in the Action Simulation Layer (ASL), where the robot internally simulates the action performed by its partner. As mention previously, action simulation and contextual information is of paramount importance in intention inference, which takes place in the Intention Layer (IL). Knowing the intention of its teammate, the object disposition (through the OML), and current state of the joint task (in the CSGL), the most appropriate complementary behaviour is selected within the Action Execution Layer (AEL). To ensure action consistency and fluency the Action Monitoring Layer (AML) continuously monitors the mismatches between inferred intention and the sub-goals that are currently possible [19]. The AML is a key factor for coping with the uncertainty of the human behaviour.

\section{A. The Dynamic Neural Field framework}

Each layer is implemented using the theoretical framework of the Dynamical Field Theory [24]-[26]. In each layer $\left(i=A S L, A E L, \ldots\right.$, , the activity $u_{i}(x, t)$ at time $t$ of a neuron at field location $x$ is described by the following integrodifferential equation [27]:

$$
\begin{aligned}
\tau_{\mathrm{i}} \frac{\delta u_{\mathrm{i}}(x, t)}{\delta t} & =-u_{\mathrm{i}}(x, t)+S_{\mathrm{i}}(x, t) \\
& +\int w_{\mathrm{i}}\left(x-x^{\prime}\right) f_{\mathrm{i}}\left(u_{\mathrm{i}}\left(x^{\prime}, t\right)\right) d x^{\prime}-h_{\mathrm{i}}
\end{aligned}
$$

These neural fields are homogeneous fields that include both excitatory and inhibitory neurons, and the temporal dynamics of individual neurons is neglected over the overall behavior of the entire population. Parameters $\tau_{\mathrm{i}}$ and $h_{\mathrm{i}}<0$ define the time scale and the resting level of the dynamic field, respectively, $S_{\mathrm{i}}$ represents the external input applied to the field at location $x$ and time $t$, and $u_{i}(x, t)$ represents the activity of a neuron coding the field location $x$ and time $t$.

By convention the dynamic neural fields (DNF) of lateralinhibition type, the excitatory connections dominate at proximal distances and the inhibitory connections dominate at greater distances [27]. The excitatory or inhibitory behavior of a neuron depends only on its distance to the activation site, $w\left(x-x^{\prime}\right)$ (eq. 1). The overall interaction behavior of all neurons can thus be modeled by a Gaussian function minus a constant value, $w_{\text {inhib }}$ :

$$
w\left(x-x^{\prime}\right)=A e^{-\frac{\left(x-x^{\prime}\right)^{2}}{2 \sigma^{2}}}-w_{i n h i b}
$$

where $A>0$ and $\sigma>0$ define, respectively, the amplitude and standard deviation, and the constant $w_{\text {inhib }}>0$ represents the global inhibition that the active neurons carry on the rest of the field. The neurons in each DNF can be either active or inactive, and only the neurons that received enough amount of input to become positively active contribute to the internal interactions of the neural field and are able to transmit information to the down-stream systems. Additionally, it is assumed that when the neurons pass over the activation threshold, they all fire at their maximum rate, regardless of the magnitude of the input pattern. To model these properties, the field dynamics must become highly nonlinear, which is achieved through the sigmoid function $(f(u))$ showed in equation 3:

$$
f(u)=\frac{1}{1+e^{-\beta\left(u-u_{0}\right)}}
$$

where $u_{0}$ is the threshold and $\beta>0$ is the slope parameter [25].

The summed input from connected fields $u_{1}$ is given as $S_{\mathrm{i}}(x, t)=k \sum_{\mathrm{l}} S_{\mathrm{l}}(x, t)$. The parameter $k$ scales the total input to a certain population relative to the threshold for triggering a self-sustained pattern. This guarantees that the inter-field couplings are weak compared to the the recurrent interactions that dominate the field dynamics (for details see [25]). The scaling also ensures that missing or delayed input from one or more connected populations will lead to a subthreshold activity distribution only. The input from each connected field $u_{1}$ is modeled by Gaussian functions

$$
S_{\mathrm{l}}(x, t)=\sum_{\mathrm{m}} \sum_{\mathrm{j}} a_{\mathrm{mj}} c_{\mathrm{l}}(t) \exp \left(-\left(x-x_{\mathrm{m}}\right)^{2} / 2 \sigma^{2}\right)
$$

where $c_{1}(t)$ is a function that signals the presence or absence of a self-stabilized activation peak in $u_{\mathrm{l}}$, and $a_{\mathrm{mj}}$ is the interfield synaptic connection between subpopulation $\mathrm{j}$ in $u_{1}$ to subpopulation $m$ in $u_{\mathrm{i}}$. Inputs from the vision system are also modeled as Gaussians for simplicity.

\section{RESUlts}

The dynamic control architecture was validated in a series of human-robot interactions under the proposed scenario. Different video snapshots are shown to illustrate the impact of action observation on complementary action selection. We will briefly show how the human actions, the action context, and even human personality can influence the robot's decision process. In these tests it is assumed that: i) the human ultimate goal is to drink; ii) the robot has prior knowledge about the task, i.e. the robot knows that first it is necessary to open the bottle and/or turn the glass in upright position before filling it; iii) the robot knows that a bottle grasping from above (above grip, AG) means that the human most likely is going to handover the object; iv) grasping the bottle from the side (side grip, SG) means that probably the human will try to pour the juice in the glass; v) grasping the glass from the side 
means that the human will likely try to invert it or to drink depending on its state. There is not a one to one mapping and in previous work we have shown that an imitation learning paradigm can be used to transfer the knowledge about this specific grip-goal relation from a human teacher to the robot that takes into account the action context [28].

\section{A. Goal Inference and Action Monitoring in Cooperative Interaction}

As mentioned previously, the robot is capable of acquiring the contextual information. Specialized pools of neurons in the OL, in the OML and in the CSGL code the motor and contextual information, and enable the robot to perform the action simulation and intention inference. The decision cycle is always composed by the action simulation and intention inference steps. These two processes allow the robot to continuously track and relate actions with their underlying context, monitoring their outcomes. Fig. 3 shows the impact of action simulation, goal inference and action monitoring on action execution, which may include overt motor behavior and/or speech. The robot monitors the behavior of the human agent, selecting the most appropriate action, taking always into account the interaction scenario constraints and the agents' physical limitations.

Due to their motor disabilities, human and robot have to cooperate to open the bottle (Panel A, snapshot S1). The human action is decomposed into its motor primitives, i.e., Reach-Grasp-Closed-Bottle with SG. This information (OL) along with the contextual information within the OML and the CSGL activates the pool of neurons coding the goal direct action-chain Reach-Grasp-SG-Closed-Bottle in ASL layer (panel B $(T 0-T 1)$ ). Given this, the robot infers the intention of removing the stopper within the IL. The robot is not capable to grasp and rotate such a small object and the control architecture produces an error in the AML. The pattern of field activation within the AML, coding that error, directly influences the action selection process in the AEL. As a result of that, the robot does not perform any motor act but verbally communicates to the human its own physical limitation (panel A, snapshots $S 2$ and $S 3$ ). As it can be seen in panel $\mathrm{C}(T 0-T 1)$, all stimuli in the AEL disappear and Communicate - Error neuronal population wins. The audio message that is displayed depends solely on the internal competition within AML.

\section{B. Action Coordination and Fluency}

The two agents have physical limitation that need to be mutually compensated. Fig. 3, snapshots $S 4$ to $S 6$ in panel A, show one example of the process of action coordination between the two agents that allow coping with their physical limitations. The human reaches and grasps the closed bottle (CB) from above. The motor action is decomposed in its elementary motor primitives, Reach - Grasp $-C B$ with Above Grip (AG) (Fig. 3 snapshots $S 4$ and $S 5$ ). This sequence of motor primitives is associated with handing over the $\mathrm{CB}$ in the ASL and the intention of removing the stopper in the IL.
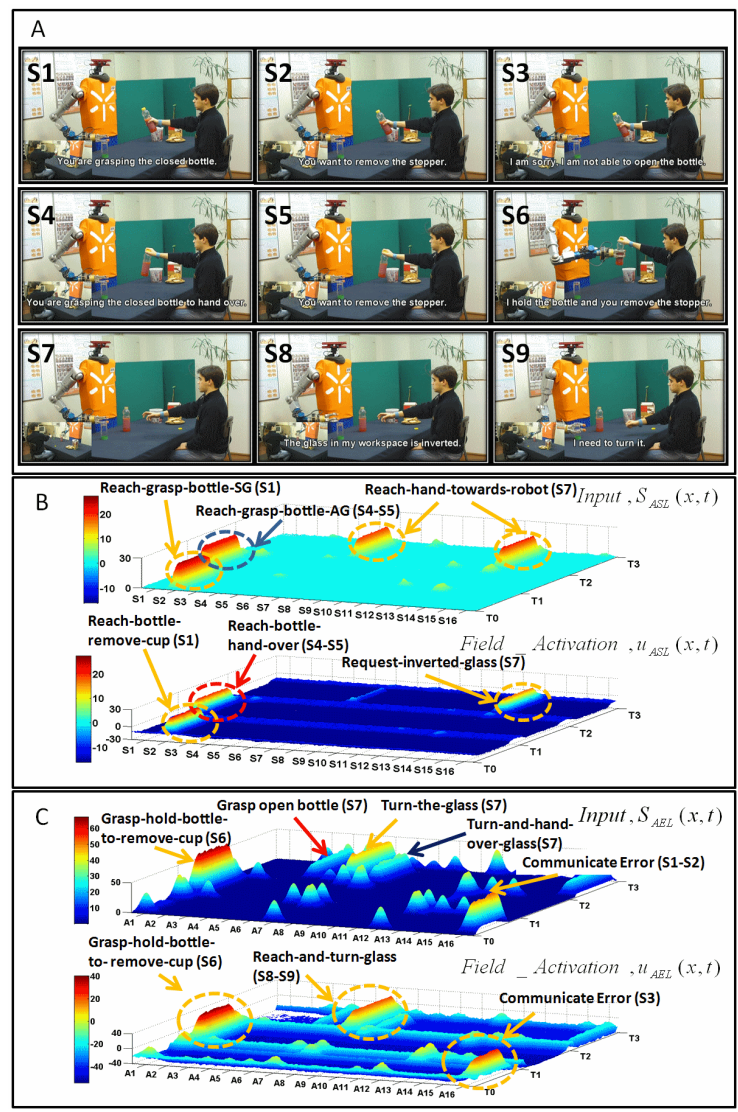

Fig. 3. An example that shows the impact of goal inference and action monitoring on the process of complementary action selection. Panel A: video snapshots. Panel B: Temporal evolution of the input (top) to ASL and activity in ASL (bottom). Panel C: Temporal evolution of the input to AEL (top) and activity in AEL (bottom).

Contrary to the previous situation (snapshots $S 1-S 3$ ), the action of grasping the bottle from above enables the robot to safely grasp and hold it out so the human can remove the stopper. The robot decides to hold the bottle for the human and gives him instructions that he/her may remove the cup (panel A, snapshot S6). This decision process does not come from a purely reactive behavior but rather comes from some level of reasoning about the interaction scenario. Snapshots $S 7$ to $S 9$ of Fig. 3 provide a good example and reveal the importance of the contextual data on the final decision. The human reaches his hand towards the robot which infers that the human is requesting the (inverted) glass in its workspace. The selection of Reach - and - turn - glass (A6) over the Reach - Turn - and - hand - over (A7) comes from the fact within the OML it is encoded an OB in the RWS. This situation evidences that in each action selection process there is always a basic reasoning mechanism. The robot has the bottle in its workspace, and it is open, it is more efficient to hand over the glass but only when filled by the robot itself.

The capability to perceive and predict the intentions underlying ongoing actions is important for fluent and efficient interaction, and is a fundamental feature to turn the robot into 
an effective socially aware assistant robot.

Fig. 4 presents two different situations in which the flexibility of the decision process is crucial for interaction consistency and fluidity. In snapshot S10 (in panel A) the robot has the Open Bottle (OB) and the Empty Glass (EG) in its workspace. Two goals: Fill - glass and Hand - over - full - glass need to be fulfilled. To ensure that the decision produced was really based on a correct understanding of the environmental state and constraints, a three layered system for the CSGL was adopted. With this layout the robot's understanding about what can be done now and what can be done in the future it is clearly represented. In panel A (Fig. 4) one can observe which goals have been met at the GSGL - Past (Open - bottle and Turn - glass sub-goals); what goals should be under the robot's attention (Fill - glass and the Hand - over full-glass sub-goals). Since it is necessary to satisfy first the Fill-glass sub-goal over the Hand-over-full-glass sub-goal, an activation peak emerges in the GSGL-Present coding that priority. The consequence of this is that the decision process is biased to produce an output that drives the robot to Reach - grasp - open - bottle - fill - glass, Fig. 4 snapshot $S 11$ and $S 12$. At snapshot $S 13$ the robot faces a similar situation and it has to decide on its own which path the interaction should take. From the panel B $(T 4-T 5)$ of Fig. 4 one can observe that now the Fill-glass sub-goal is at the GSGL-Past and the Hand-over-full-glass in the $G S G L-$ Present. The robot's now reaches the full glass and hands it over to the human (Fig. 4 snapshot $S 14$ and $S 15$ and Reach - full - glass - to - hand-over from panel C $(T 4-T 5))$. These two situations show that the CSGL play a very important role in flexible action selection.

\section{User Adaptation}

To assess adaptation to different users, experiments were carried with humans with different attitude. One of these interaction scenarios was composed by a CB and an EG in the upright position. The sub-goal of turning the glass is, at the outset of the interaction, accomplished (see Fig. 5, panel B $(T 0-T 1) C S G L-$ Past). From the $C S G L-$ Present the need of opening the bottle is extracted and the decision in AEL is biased toward the robot asking for help to open the bottle. On the AEL emerges the action of Grasp-holdbottle-to-remove-cup panel C $(T 0-T 1)$ (snapshots $S 1$ to $S 3$ in panel A). The acquired contextual information enables the robot to update the objects state and the Open - bottle sub-goal vanishes from the CSGL - Present and emerges at the $C S G L-$ Past and the Fill-glass previously at the $G S G L-F u t u r e$ rises at the $C S G L-$ Present, setting the next interaction priority (panel B $(T 1-T 2) C S G L-$ Present and $C S G L-$ Future). Next the robot has the $\mathrm{OB}$ in the RWS and the EG remains at the HWS. In this situation the robot has two possible actions competing, pass the bottle to the human or ask for the glass (panel $\mathrm{C},(T 1-T 2)$ ). It decides to request the glass and reaches its hand towards the human (panel A, snapshots $S 4$ to $S 6$ ). The goal of filling the glass was not yet satisfied, and this task sub-goal remains

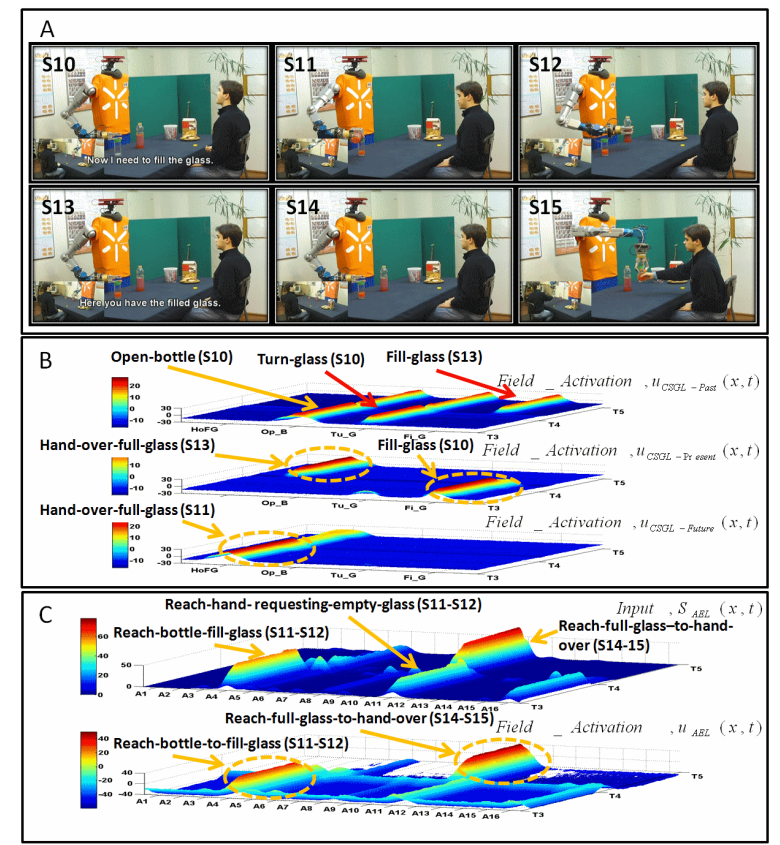

Fig. 4. The impact of CSGL in anticipatory action selection. Panel A: continuation of the snapshots of video in Fig. 3. Panel B: Field activity in CSGL Past, Present and Furure layers, respectively. Panel C:Temporal evolution of the input to AEL (top) and activity in AEL (bottom).

active from the current interaction to the next one (panel $\mathrm{B},(T 2-T 3) C S G L-$ Present $)$. At the time between $T 2$ and $T 3$ the robot fills the EG in its workplace (panel A, snapshots $S 7$ to $S 9$ ), the Fill - glass sub-goal disappears from the $C S G L-$ Present and rises at the CSGL - Past and then the Hand-over-full-glass is set as the present sub-goal (panel B (T3-T4) CSGL-Present).

\section{CONCLUSION}

In order to interact with humans in a social context, robots must be capable of extracting meaning from the environment. In the proposed interaction context, the robot had to extract information from the scene and also from its partner, by tracking his/her goal directed actions, their context, and infer the underlying motor intentions. The Cognitive Architecture presented has implemented the elementary features of the MNS, in an attempt to endow the robot with rudiments of action understanding and goal inference. By representing the action in its' cognitive structure, the robot was able to extract their underlying action intentions. Clear evidences of this are the action monitoring and flexible action allocation abilities demonstrated by the robot. Intention inference seems to be a fundamental mechanism for robust and adequate complementary action selection, and for bias interaction fluency. The robot has also demonstrated behaviours far more complex than a purely reactive agent. It was capable of seen beyond the human's desires and perform actions that were in fact a step forward in the interaction, Fig. 3 snapshots $S 7$ to $S 9$.

In addition, the use the nonverbal communication provides a common ground of understanding between teammates and 


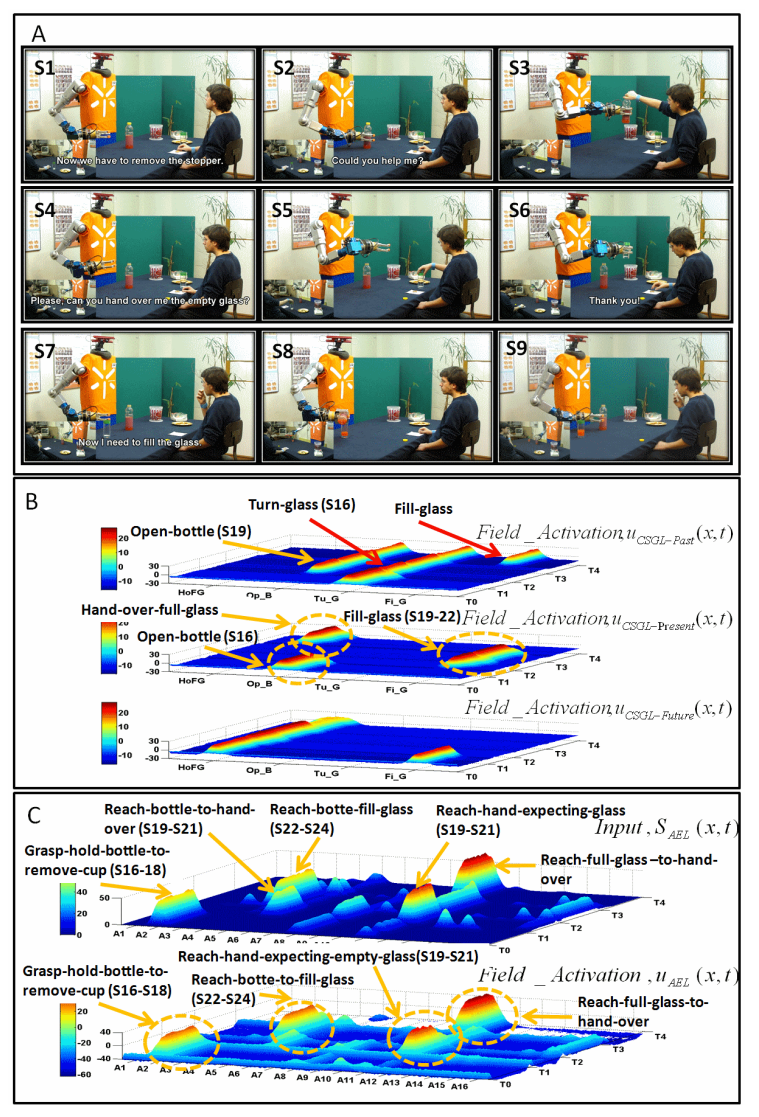

Fig. 5. An example that illustrates the capability of the robot to take the initiative when interacting with a passive person. Panel A: Video snapshots. Panel B: Field activity in CSGL Past, Present and Furure layers, respectively. Panel C: Temporal evolution of the input to AEL (top) and activity in AEL (bottom).

enables the robot to interact with people from different cultural background and geographic areas. Moreover, the results show the importance of the OML and CSGL in user adaptation and ability establish interaction. The robot was able to engage in interaction with the human even when he/she was more passive or totally passive. This feature is of paramount importance if one considers the application of robots as socially assistive agents in healthcare facilities or in home environments, providing additional care to motor of cognitive disabled people.

\section{ACKNOWLEDGMENT}

The authors would like to thank Eliana Silva, Emanuel Sousa, Flora Ferreira, João Ferreira, Joaquim Silva, Luís Louro, Nzoji Hipolito, Rui Silva, Tiago Malheiro and Toni Machado.

\section{REFERENCES}

[1] T. Fong, I. Nourbakhsh and K. Dautenhahn, "A survey of socially interactive robots," Robotics and autonomous systems, vol. 42, no. 3, pp. 143-166, 2003.

[2] C. Breazeal, "Social interactions in HRI: the robot view," IEEE Transactions on Systems, Man, and Cybernetics, Part C: Applications and Reviews, vol. 34 , no. 2, pp. 181-186, 2004.

[3] S. Schaal, "The new robotics-towards human-centered machines," HFSP journal, vol. 1, no. 2, pp. 115-126, 2007.
[4] K. Dautenhahn and K. Dautenhahn, "Socially intelligent robots: dimensions of human-robot interaction," Philosophical Transactions of the Royal Society B: Biological Sciences, vol. 362, no. 1480, pp. 679-704, 2007.

[5] A. Tapus, M. J. Mataric, B. Scasselati, K. Dautenhahn and K. Dautenhahn, "Socially assistive robotics [Grand challenges of robotics]," IEEE Robotics \& Automation Magazine, vol. 14, no. 1, pp. 35-42, 2007.

[6] B. Reeves and C. I. Nass, The media equation: How people treat computers, television, and new media like real people and places, Cambridge, UK: Cambridge University Press, 1996.

[7] N. Sebanz, H. Bekkering and G. Knoblich, "Joint action: bodies and minds moving together," Trends in cognitive sciences, vol. 10, no. 2, pp. 70-76, 2006.

[8] R. D. Newman-Norlund, M. L. Noordzij, R. G. J. Meulenbroek and H. Bekkering, "Exploring the brain basis of joint action: Co-ordination of actions, goals and intentions," Social neuroscience, vol. 2, no. 1, pp. 48-65, 2007.

[9] C. Breazeal, C. D. Kidd, A. L. Thomaz, G. Hoffman and M. Berlin, "Effects of nonverbal communication on efficiency and robustness in human-robot teamwork," IEEE/RSJ International Conference on Intelligent Robots and Systems (IROS 2005), 2005.

[10] Wilson, M. and Knoblich, G., "The case for motor involvement in perceiving conspecifics," Psychological bulletin, vol. 131, pp. 460, 2005

[11] L. Fogassi, P. F. Ferrari, B. Gesierich, S. Rozzi, F. Chersi and G. Rizzolatti, "Parietal lobe: from action organization to intention understanding," Science, vol. 308, no. 5722, pp. 662-667, 2005.

[12] R. D. Newman-Norlund, H. T. van Schie, A. M. J. van Zuijlen and H. Bekkering, "The mirror neuron system is more active during complementary compared with imitative action," Nature neuroscience, vol. 10, no. 7, pp. 817-818, 2007

[13] W. Erlhagen, A. Mukovskiy and E. Bicho, "A dynamic model for action understanding and goal-directed imitation," Brain research, vol. 1083, no. 1, pp. 174-188, 2006.

[14] H. Bekkering, E. R. A. De Bruijn, R. H. Cuijpers, R. Newman-Norlund, H. T. Van Schie and R. Meulenbroek, "Joint action: Neurocognitive mechanisms supporting human interaction," Topics in Cognitive Science, vol. 1, no. 2, pp. 340-352, 2009.

[15] G. Rizzolatti, L. Fogassi and V. Gallese, "Neurophysiological mechanisms underlying the understanding and imitation of action," Nature Reviews Neuroscience, vol. 2, no. 9, pp. 661-669, 2001.

[16] G. Rizzolatti and L. Craighero, "The mirror-neuron system," Annu. Rev. Neurosci., vol. 27, pp. 169-192, 2004.

[17] V. Gazzola, G. Rizzolatti, B. Wicker and C. Keysers, "The anthropomorphic brain: the mirror neuron system responds to human and robotic actions," Neuroimage, vol. 35, no. 4, pp. 1674-1684, 2007.

[18] M. Iacoboni, I. Molnar-Szakacs, V. Gallese, G. Buccino, J. C. Mazziotta and G. Rizzolatti, "Grasping the intentions of others with one's own mirror neuron system," PLoS biology, vol. 3, no. 3, pp. e79, 2005.

[19] E. Bicho, L. Louro, N. Hipólito and W. Erlhagen, "A dynamic field approach to goal inference and error monitoring for human-robot interaction," Proceedings of the 2009 International Symposium on New Frontiers in Human-Robot Interaction, AISB 2009 Convention, HeriotWatt University Edinburgh, pp. 31-37, 2009.

[20] E. Bicho, W. Erlhagen, L. Louro and E. Costa e Silva, "Neurocognitive mechanisms of decision making in joint action: A human-robot interaction study," Human movement science, vol. 30, no. 5, pp. 846-868, 2011.

[21] R. Silva, E. Bicho and W. Erlhagen, "Aros: An anthropomorphic robot for human-robot interaction and coordination studies," Proc. of the Portuguese $8^{\text {th }}$ Conference on Automatic Control-Controlo, pp. 819-826, 2008.

[22] G. Westphal, C. Von Der Malsburg and R. Würtz, "Feature-driven emergence of model graphs for object recognition and categorization," Applied Pattern Recognition, pp. 155-199, 2008.

[23] E. Costa e Silva, M. F. Costa, E. Bicho and W. Erlhagen, "HumanLike Movement of an Anthropomorphic Robot: Problem Revisited," AIP Conference Proceedings, vol. 1389, pp. 779-782, 2011.

[24] J. P. Spencer and G. Schöner, "Bridging the representational gap in the dynamic systems approach to development," Developmental Science, vol 6, no. 4, pp. 392-412, 2003.

[25] W. Erlhagen and E. Bicho, "The dynamic neural field approach to cognitive robotics," Journal of Neural Engineering, vol. 3, no. 3, pp. R36, 2006. 
[26] G. Schöner, "Dynamical systems approaches to cognition," in Cambridge handbook of computational cognitive modeling, Cambridge, UK: Cambridge University Press, 2008, pp. 101-126.

[27] S. Amari, "Dynamics of pattern formation in lateral-inhibition type neural fields," Biological cybernetics, vol. 27, no. 2, pp. 77-87, 1977.

[28] W. Erlhagen, A. Mukovskiy, E. Bicho, G. Panin, C. Kiss, A. Knoll, H. Van Schie and H. Bekkering, "Goal-directed imitation for robots: A bio-inspired approach to action understanding and skill learning," Robotics and Autonomous Systems, vol. 54, no. 5, pp. 353-360, 2006. 